\title{
CONTRIBUtion A L'ÉTude du DIACÉTYLE
}

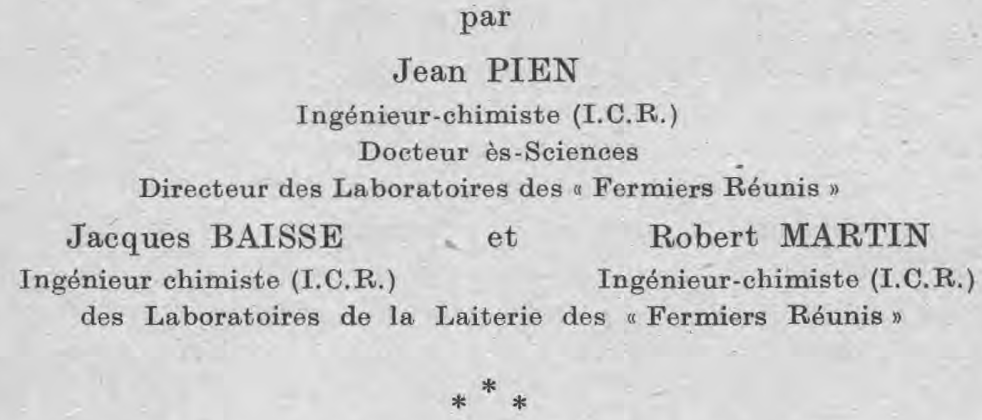

La loi du 2 juillet 1935, sur l'assainissement du marché du lait, a réglé d'une manière définitive la question de l'emploi du diacétyle dans la préparation du beurre. L'utilisation de cet " arôme ", son incorporation à la crème ou au beurre sont interdites.

Est-ce à dire que l'étude chimique et biologique de cette substance doive être abandonnée ? Certainement pas. De nombreuses raisons, au contraire, nous font penser que de tels travaux doivent être poursuivis.

L'étude chimique de la fermentation lactique exige la connaissance de la constitution, des propriétés, des conditions de formation d'un certain nombre de substances qui constituent des termes de passage au cours de la transformation du lactose en acide lactique ou qui apparaissent à côté d'elles dans les fermentations lactiques non spécifiques. La biologie des ferments lactiques pose les mêmes problèmes; ce n'est là, d'ailleurs, qu'un cas particulier de ces disciplines nouvelles de la bactériologie moderne qui réclament de plus en plus le concours de la chimie.

Mais il y a d'autres raisons de poursuivre l'étude du diacétyle : la loi en interdit l'usage ; il faut pouvoir contrôler l'application de la loi. Il faut pouvoir apporter des documents nombreux sur les taux de diacétyle naturel contenu dans certains beurres. II faut pouvoir le doser avec certitude dans les beurres suspects d'avoir été "enrichis". L'étude des conditions de formation du diacétyle naturel, la détermination des maxima possibles dans certaines eultures, etc., permettront d'orienter et d'interpréter des recherches particulières.

De toute manière, nous pensons que les progrès réalisés dans la recherche et le dosage du diacétyle faciliteront l'application de la loi et réaliseront de ce fait une meilleure protection de l'industriel honnête contre la coneurrence des fraudeurs.

$$
\text { *** }
$$


Le très intéressant travail que M. TAPERnoux a fait paraître ici même (Le Lait, décembre 1932, p. 1.043) a déjà rassemblé, sur cette question, un certain nombre de documents importants.

Toutefois, à l'usage, la méthode de dosage proposée par M. TAPERnodx (méthode de Lemoigne, par formation de diméthylglyoximate de nickel) ne nous a pas paru posséder la sensibilité et la régularité requises d'une bonne méthode d'analyse quantitative. Dans un domaine aussi délicat que celui de l'anplieation technique d'une loi, il faut de très sérieuses garanties. Nous aurons l'oceasion de nous expliquer plus en détail à ce sujet. Nous avons eru devoir mettre au point une méthode de dosage plus sensible et surtout d'une plus grande régularité. Nous pensons y avoir réussi.

Nous avons cru bon également d'étudier en détail la préparation du diacétyle (par la voie chimique) afin de partir de matériaux de composition parfaitement connue et définie pour le contrôle des procédés de dosage.

Enfin, nantis de ces possibilités de contrôle, nous avons poussé un peu plus loin notre étude et avons cherché à faire ouvre pratique en dosant le diacétyle dans des crèmes, des beurres, dans des cultures de ferments variés, etc., c'est-à-dire en fournissant des documents qui peuvent, un jour ou l'autre, avoir leur utilité.

Notre plan sera done le suivant :

10 Etude de la préparation chimique du diacétyle.

$2^{\circ}$ Recherche qualitative et identification du diacétyle.

$3^{\circ}$ Nouveau procédé de dosage du diacétyle.

$4^{0}$ Application à l'étude de la crème, du beurre, des eultures pures de ferments lactiques, etc.

$5^{0}$ Discussion sur l'interdiction de l'emploi du diacétyle et conclusions.$$
*^{*} *
$$ \\ PREMIERE PARTIE}

\section{ETUDE DE LA PRÉPARATION DU DIACÉTYLE}

Nous ne relaterons pas les différents essais auxquels nous avons procédé au cours de ce travail. Nous avons étudié, en effet, plusieurs modes de préparation, mais nous nous sommes finalement arrêtés à la méthode qui donne le meilleur rendement en diacétyle, et qui est en même temps la moins dangereuse pour l'opérateur.

\section{Principe de la méthode adoptée.}

Le but à atteindre est la préparation d'un corps de formule :

$$
\mathrm{CH}^{3}-\mathrm{CO}-\mathrm{CO}-\mathrm{CH}^{3}
$$

c'est-à-dire une dicétone $\alpha$. 
La chimie organique enseigne plusieurs méthodes pour former des dicétones où les deux $\mathrm{CO}$ sont contigus.

Le procédé que nous avons choisi part d'une cétone ayant le même nombre d'atomes de carbone que le diacétyle. Il est en effet souvent plus difficile de faire entrer un carbone nouveau dans une molécule, que de transformer un groupement $\mathrm{CX}^{\mathrm{n}}$ en un groupement cétonique $\mathrm{CO}$.

Nous partirons done, non pas de l'acétone $\mathrm{CH}^{3}-\mathrm{CO}-\mathrm{CH}^{3}$ qui n'a que trois carbones, mais de son homologue supérieur : la méthylacétone ou méthyléthylcétone de formule :

$$
\mathrm{CH}^{3}-\mathrm{CO}-\mathrm{CH}^{2}-\mathrm{CH}^{3}
$$

Le problème qui se pose consiste donc à transformer un $\mathrm{CH}^{2}$ en un CO. C'est un cas classique que la chimie organique sait très bien résoudre mais qui présente une difficulté particulière du fait de la présence dans la molécule d'un groupe cétonique à côté du $\mathrm{CH}^{2}$ à transformer.

On prendra done soin de bloquer d'abord le CO déjà existant par une nitrosation; et, après transformation du $\mathrm{CH}^{2}$ en $\mathrm{CO}$, on régénérera le premier groupement cétonique qui avait été bloqué.

D'où le principe de la technique à employer :

On part de la méthyléthylcétone $\mathrm{CH}^{3}-\mathrm{CO}-\mathrm{CH}^{2}-\mathrm{CH}^{3}$ que l'on nitrose à l'aide du nitrite d'amyle. Il se forme de ce fait un composé qui est la nitrosométhyléthylcétone (où le groupement cétonique est bloqué) de formule :<smiles>CC(C=O)=NO</smiles>

Par action de la soude étendue sur cette substance, on forme l'isonitrosométhyléthylcétone ou monoxime du diacétyle (le $\mathrm{CH}^{2}$ se trouve remplacé par un $\mathrm{CO}$ ).<smiles></smiles>

Enfin, par ébullition avec l'acide sulfurique étendu, la monoxime libère le diacétyle par régénération du premier $\mathrm{CO}$ :

$$
\mathrm{CH}^{3}-\mathrm{CO}-\mathrm{CO}-\mathrm{CH}^{3}
$$

\section{Mode opératoire.}

Nous nous sommes inspirés des indications données dans le traité "Méthodes de la chimie organique) de WEYL (tome III, p. 432). Nous avons dû modifier ces indications pour les adapter à 
nos besoins et aussi pour nous permettre de préparer une quantité suffisante de diacétyle. ( $\mathrm{Si}$ on se borne à travailler sur de trop petites quantités de matières le rendement obtenu est insignifiant.)

L'opération comporte trois stades :

10 Nitrosation de la méthyléthylcétone et isolement de l'isonitrosométhyléthylcétone.

$2^{\circ}$ Hydrolyse de ce produit et obtention de diacétyle brut.

$3^{\circ}$ Purification et rectification du diacétyle.

\section{Premier Temps.}

$850 \mathrm{cc}$ de méthyléthylcétone sont additionnés de $30 \mathrm{cc}$ d'acide chlorhydrique pur (densité 1,19). On ajoute à ce mélange, petit à petit et en refroidissant, constamment 1 litre de nitrite d'amyle. Il faut bien veiller à ne pas dépasser la température de $50^{\circ}$ au sein du liquide : si la température s'élevait trop, on formerait des produits nitrés, ce qu'il faut éviter.

L'ensemble de l'opération de nitrosation conduite sur ces bases demande environ une heure.

Ajouter alors 800 grammes de glace et 600 ce de lessive de soude à $33 \%$. Agiter violemment, puis laisser en contact une heure.

Ensuite décanter, recueillir la partie aqueuse sous-jacente et la débarrasser de l'excès d'alcool amylique (libéré par la réaction) en l'agitant deux fois consécutives (suivies de décantation) avec un égal volume d'éther sulfurique.

Cette partie aqueuse est à nouveau additionnée de $1 \mathrm{~kg}$. de glace et neutralisée progressivement avec de l'acide sulfurique au demi. Il se précipite de l'isonitrosométhyléthylcétone ou monoxime du diacétyle. Laisser reposer une heure à température aussi basse que possible, puis filtrer au Büchner et presser.

\section{Deuxième Temps.}

Introduire le gâteau de diacétyloxime dans un ballon de 10 litres avec 4 litres d'eau et 500 ce d'acide sulfurique à $66^{\circ}$ Bé. Agiter et entraîner à la vapeur d'eau.

Il est nécessaire d'installer bout à bout trois réfirigérants de Vigreux de 1 mètre de long chacun afin d'assurer une réfrigération énergique et éviter les pertes de diacétyle.

Cette opération fournit un litre à 1 litre $1 / 2$ d'un liquide jaune qui contient le diacétyle dilué et impur. (On arrête la distillation quand une goutte de distillat ne donne plus la réaction de Tchugaïeff au diméthylglyoximate de nickel.)

Ce distillat est à nouveau repris par entraînement à la vapeur de manière à concentrer le liquide en diacétyle.

On ajoute au distillat $30 \%$ en poids de chlorure de sodium, 
on effectue un troisième entraînement à la vapeur et on recueille un nouveau distillat dans une ampoule à décantation.

Le diacétyle se rássemble à la surface en une couche huileuse que l'on sépare.

(La couche sous-jacente est conservée et redistillée à nouveau pour concentrer et extraire le diacétyle qu'elle retient en solution.)

\section{Troisième Temps.}

Le diacétyle impur ainsi obtenu est déshydraté par addition de Ca $\mathrm{Cl}^{2}$ desséché.

On procéde ensuite à une rectification très soignée dans une colonne de Robert. On ne recueille que la portion passant exactement à $88^{\circ}$.

(Les premières portions du distillat sont constituées par de la méthyléthylcétone, et les queues par de l'aleool amylique.)

Le diacétyle ainsi obtenu est pratiquement pur.

Remarques : $1^{\circ}$ Pour avoir un diacétyle rigoureusement pur $100 \%$, on fera agir (sur le produit déjà rectifié) la phénylhydrazine de manière à former une osazone. Cette osazone, purifiée par cristallisations successives, sera ensuite distillée avec de l'acide sulfurique étendu et on recusille ra du diacétyle pur qu'on achèvera de dessécher sur $\mathrm{Ca} \mathrm{Cl}^{2}$.

20 Rendement : Le rendement de l'opération est très faible lorsqu'on travaille sur de petites quantités à cause du grand nombre de manipulations successives. En opérant comme nous l'avons exposé ci-dessus, nous obtenons un rendement moyen de 12 à $15 \%$ du poids de la méthyléthylcétone mise en œuvre. Pratiquement, en opérant avec les quantités indiquées, on obtient 150 à $200 \mathrm{ce}$ de diacétyle brut et seulement $100 \mathrm{cc}$ (environ) de diacétyle pur.

$3^{\circ}$ Conservation du diacétyle. - Le diacétyle est introduit dans des ampoules de verre qu'on scelle à la flamme et que l'on conserve à l'obscurité. Il faut, en effet, éviter le contact de l'air et surtout l'action de la lumière.

Même en prenant ces précautions il arrive que des échantillons s'altèrent, bien que correctement préparés. Cela tient à ce que certains verres provoquent, avec le temps, une modification du produit. Il peut être intéressant alors de conserver le diacétyle dans un récipient métallique (aluminium).

Néanmoins nous avons réussi, en employant des ampoules de verre bien neutre, à conserver sans altération du diacétyle pur, sous réserve qu'il ait été très soigneusement rectifié. 


\section{DEUXIËME PARTIE}

\section{RECHERCHES QUALITATIVES ET IDENTIFICATION DU DIACETYLE}

Jusqu'à présent on s'est contenté, dans la pratique courante, de faire appel à la formation de diméthylglyoximate de nickel.

On sait que les aldéhydes et les cétones peuvent réagir avec l'hydroxylamine $\mathrm{NH}^{2} \mathrm{OH}$ et donner (avec élimination d'eau) des corps cristallisés qu'on appelle des oximes (aldoximes et cétoximes). 5n particulier le glyoxal ou dialdéhyde $\mathrm{CHO}-\mathrm{CHO}$ réagit avec deux molécules d'hydroxylamine pour donner l'aldoxime correspondante ou glyoxime.

On peut écrire :

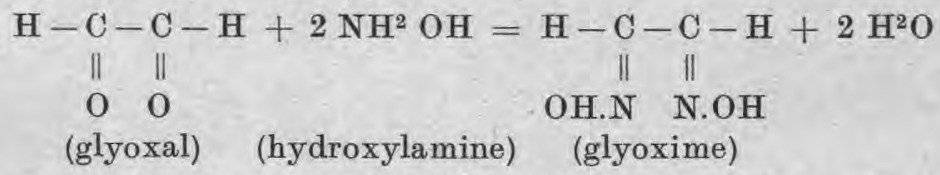

Prenons de même le cas du diacétyle. Ce corps $\mathrm{CH}^{3}-\mathrm{CO}-\mathrm{CO}$ - $\mathrm{CH}^{3}$ peut être considéré comme le dérivé diméthylé du glyoxal. D'ailleurs, on peut, correctement, l'appeler : diméthylglyoxal.

Répétons l'opération précédente. Nous aurons cette fois formation d'une glyoxime diméthylée ou diméthylglyoxime suivant la réaction :

$$
\begin{aligned}
& \mathrm{CH}^{2}-\mathrm{C}-\mathrm{C}-\mathrm{CH}^{3}+2 \mathrm{NH}^{2} \mathrm{OH}=\mathrm{CH}^{3}-\mathrm{C}-\mathrm{C}-\mathrm{CH}^{3} \\
& \text { O } 0 \\
& \text { (diacétyle) } \\
& \text { (hydroxylamine) }
\end{aligned}
$$

Or on sait depuis longtemps que les glyoximes forment avec certains sels métalliques des combinaisons colorées, bien cristallisées, peu solubles. En particulier on connaît, sous le nom de réaction de Tchugaïeff, le moyen d'identifier et de doser le nickel par formation du diméthylglyoximate de nickel. Cette réaction est couramment employée et donne de bons résultats pour le dosage de ce métal.

On a done voulu réaliser l'opération inverse et utiliser les sels de nickel pour doser la diméthylglyoxime (provenant elle-même du diacétyle que l'on transforme).

Les conditions de la réaction initiale sont donc complètement modifiées.

Au lieu d'avoir un excès d'oxime, comme c'est le cas dans la réaction de Tchugaïeff, on a ici un excès de nickel. En modifiant les eirconstances de la réaction on parvient néanmoins à en faire un procédé très acceptable, au moins au point de vue qualitatif. M. Lemoigne en a même tiré une méthode de dosage de diacétyle - rappelée par M. TAPERNOUX dans son article. 
Nous avons expérimenté cette méthode.

Nous croyons pouvoir lui faire deux reproches:

$1^{0}$ Même en suivant scrupuleusement les indications de l'auteur, on ne parvient pas toujours à une absolue régularité. Des dosages effectués en double ou en triple sur des solutions contenant la même dose de diacétyle pur, ne conduisent pas toujours au même résultat.

Il semble que des facteurs impondérables (tels que température, $\mathrm{pH}$ du milieu, etc.) jouent un rôle un peu perturbateur dont on n'est pas absolument maître, sur la solubilité du complexe métallique.

Ce n'est là qu'une hypothèse; mais il y a d'autres exemples en chimie analytique d'ineertitudes de cet ordre.

Les complexes métalliques (et à fortiori les organométalliques), quand ils sont utilisés en tant que précipités et non en tant que matières colorées solubles, soulèvent de graves problèmes de solubilité parfois difficiles à résoudre.

On a pu dans certains cas parvenir à des mises au point satisfaisantes (dans le cas des complexes du platine pour le dosage du potassium par exemple). Mais il reste souvent des incertitudes qui portent soit sur la solubilité (cas des complexes du sodium par exemple) soit sur la composition du précipité (cas des phosphomolybdates) ou sur ces deux facteurs à la fois (cas du eobaltinitrite de potassium par exemple).

Les irrégularités dans le dosage du diacétyle par le complexe de nickel ne sont donc pas exceptionnelles, ni surprenantes. Bien au contraire, nous pensions, avant même d'en faire l'applieation, qu'il serait probablement difficile dans l'état actuel de la question d'avoir par ce moyen un excellent procédé de dosage. Voici pourquoi : dans un dosage deux éléments essentiels sont en présence : le corps à doser et une deuxième substance qui s'unit à la première (ou modifie la première) pour aboutir à un précipité, à une combinaison eolorée, eto... qui constituent la matière même de la pesée, du titrage, etc...

Les conditions précises du dosage du corps No 1 par le "réactif " (corps $\mathrm{N}^{\circ} 2$ ) sont fixées par des conditions physiques et aussi par des substances accessoires dont la présence est souvent indispensable à la réussite de l'opération.

Or les chimistes ont eu souvent l'occasion de constater que si le dosage du corps $\mathrm{N}^{\circ} 1$ par le corps No 2 est au point et donne de bons résultats, il n'en est souvent plus de même quand on renverse les rôles et qu'on veut doser le corps $\mathrm{N}^{\circ} 2$ en utilisant le corps $\mathrm{N}^{\circ} 1$ comme réactif.

Exemple : le dosage du phosphore par la liqueur magnésienne est une opération facile. Combien il est difficile en revanche de faire un dosage exact de magnésium par un phosphate alealin pris. 
comme réactif de précipitation! Tel serait aussi le cas, selon nous, du dosage du nickel par la diméthylglyoxime et du dosage inverse : diacétyle (transformé en diméthylglyoxime) par le nickel.

Et eependant dans les deux cas on aboutit à une même substance dont la composition est ou doit être bien définie.

La difficulté vient de ce que, dans ce que nous appellerons le " dosage inverse ", les conditions de la réaction fondamentale sont changées. Un dosage, en effet, ne consiste pas tant à chercher la précipitation du corps $\mathrm{N}^{\circ} 1$ par le corps $\mathrm{N}^{\circ} 2$ (ou l'inverse) qu'à chercher les conditions optima de préparation du corps mixte $1+2$, but pratique du dosage. Or ces conditions optima sont généralement rigoureuses et ne peuvent guère être multiples. Si, en particulier, l'une de ces conditions exige absolument dans le dosage du corps $N^{\circ} 1$ la présence d'un grand excès du précipitant (corps $N^{\circ} 2$ ), il est clair qu'il sera bien difficile d'obtenir un dosage correct du corps No 2 en utilisant le corps $\mathrm{N}^{\circ} 1$ comme réactif.

Heureusement cette circonstance n'est pas toujours exigible, et d'ailleurs, on a des moyens de modifier favorablement l'équilibre de la réaction en faisant intervenir soit les substances accessoires dont nous parlions ci-dessus, soit des facteurs physiques. C'est la raison pour laquelle ces " dosages inverses » restent possibles.

Toutefois, et c'est là où nous voulons en venir, on conçoit aisément que l'étude du "dosage inverse" nécessite la fixation de eonditions précises, différentes de celle du " dosage direct». On peut même penser que dans certains cas le problème doit être absolument impossible à résoudre correctement.

Toutes ces raisons pourraient expliquer, selon nous, les irrégularités que nous avons constatées dans le dosage du diacétyle par le nickel. Si dans certains cas ces irrégularités peuvent ne pas être gênantes, nous avons la conviction qu'elles s'opposent, du moins dans l'état actuel de cette technique, au dosage précis de faibles quantités de diacétyle telles qu'il s'en présente dans certaines cultures.

$2^{\circ}$ Nous avons un deuxième reproche à faire à cette méthode. Il est' également grave, car il concerne la sensibilité de la réaction.

Certes, le diméthylglyoximate de nickel est un sel bien cristallisé, fortement coloré en rouge, et très insoluble.

Mais si la solution de diacétyle est diluée, la formation de ce sel est retardée ou compromise.

A la dilution de $1 / 2.000$ le précipité obtenu est très faible et très long à se produire. A la dilution de 1/5.000 la réaction ne se produit qu'en 24 heures et on n'obtient qu'une très faible quantité de cristaux microscopiques. La récolte et la pesée de ces cristaux sont impossibles. 
A des dilutions plus grandes on n'obtient absolument rien.

Or, d'une part, l'expérience nous a montré qu'il était souvent nécessaire d'opérer sur des solutions diluées, et d'autre part, la limite de la réaction se trouvant être pratiquement à $1 / 2.000$ on n'a pas une sécurité suffisante pour les récoltes et pesées de précipités provenant de solutions dont la dilution est comprise entre 1/2.000 et 1/500 par exemple.

Nous touchons là, d'ailleurs, à un inconvénient fondamental des méthodes analytiques qui font intervenir la formation de précipités dans le cas de dosages de petites quantités de matière. On s'en tire quelquefois en faisant entrer la trace de matière à doser dans une très grosse molécule correspondant à des précipités abondants (e'est le cas du dosage des traces d'urée par le Xanthydrol). Mais la chose n'est pas toujours possible, ni d'ailleurs toujours recommandable.

Quand on dose de petites quantités de matière il ne faudrait done pas avoir à former de précipités (à moins de faire appel aux méthodes de la micro-chimie). Il faut, quand la chose est possible, provoquer la formation de matières colorées, définies avec précision et les doser par colorimétrie. La légère incertitude inhérente aux méthodes colorimétriques est inférieure aux erreurs commises dans la récolte et la pesée de précipités trop faibles, et est d'ailleurs largement compensée par la possibilité de sensibilités beaucoup plus grandes.

Toute cette discussion nous amène à la conclusion que voici :

La méthode au diméthylglyoximate de nickel est une bonne méthode d'identification du diacétyle (l'aspect microscopique des cristaux, leur couleur, sont des caractères bien définis). Donc : méthode d'examen qualitatif à conserver.

Mais nous ne pensons pas que ce soit une excellente méthode de dosage car, d'une part́, elle ne paraît pas posséder une régularité rigoureuse et, d'autre part, elle n'est pas applicable à l'appréciation précise de petites quantités de diacétyle.

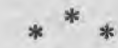

Nous avons done étudié d'autres propriétés du diacétyle en vue de découvrir des réactions capables de fournir une bonne méthode de dosage.

Nous étudierons ci-après la méthode que nous avons imaginée et mise au point.

Dans le présent chapitre, consacré à la recherche qualitative et à l'identification du diacétyle, contentons-nous de décrire une réaction colorimétrique que nous n'avons pas retenue pour le dosage, mais 
qui peut rendre de grands services au point de vue qualitatif, étant donné sa sensibilité et sa grande simplicité.

\section{RECHERCHE DU DIACÉTYLE PAR FORMATION DE XYLOQUINONE.}

On sait que les dicétones $\alpha$ peuvent, par condensation de deux molécules en milieu alcalin avec élimination d'eau, former des chaînes fermées (cyclisation) conduisant à des quinones qui sont souvent colorées.

Nous avons pensé que le diacétyle fournirait de cette manière une matière colorée quinonique. Nous avons effectivement obtenu un produit de réaction jaune-brun fluorescent.

On peut concevoir la réaction de cette manière :

$$
\begin{aligned}
2 \mathrm{CH}^{3}-\mathrm{CO}-\mathrm{CO}-\mathrm{CH}^{3} \rightarrow 2 \mathrm{H}^{2} \mathrm{O}+\mathrm{CH}^{3}-\mathrm{C}-\mathrm{CO}-\mathrm{CH} \\
\mathrm{HC}-\mathrm{CO}-\mathrm{C}-\mathrm{CH}^{3}
\end{aligned}
$$

qu'on peut encore écrire à l'aide de l'hexagone classique :

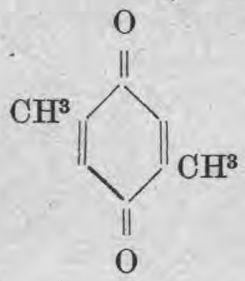

Ce schéma traduit mieux l'aspect quinonique de la substance. En fait, on a réalisé de cette manière la quinone du xylène ou xyloquinone.

Sans entrer dans le détail, voici comment nous réalisons cette réaction :

Dans un tube à essai on verse $5 \grave{a} 10 \mathrm{cc}$ de la solution contenant du diacétyle; on ajoute une pastille de potasse. On chauffe jusqu'à ébullition. Il se forme (en présence de diacétyle) une coloration jaune-brun stable même après ébullition prolongée.

Cette réaction est encore tiès nette avec une solution aqueuse de diacétyle au 1/20.000.

Objection : De nombreux corps donnent des colorations jaune-brun quand on les chauffe avec de la potasse (les sucres en particulier).

Donc cette réaction n'est pas spécifique et ne peut être utilisée avec sécurité qu'en présence de diacétyle pur.

Réponse : L'objection précédente est parfaitement fondée. Mais la recherche du diacétyle ne s'effectue jamais que sur des 
distillats ou des entraînements à la vapeur. Or, dans ces circonstances, aucun sucre, aucune matière organique ne passe (sauf bien entendu celles qui seraient volatiles ou entraînables).

En fait, un distillat de lait (exempt de diacétyle au départ) ne donne aucune réaction colorée. Donc le lait n'abandonne rien qui puisse faire croire à la présence de diacétyle. Bien entendu, s'il s'était produit un entraînement de mousses pendant la distillation, on aurait une réaction rappelant celle du diacétyle. Mais cet entrấnement ne peut pas passer inaperçu et il est facile d'y remédier par une deuxième distillation.

En fait, nous n'avons jamais été gênés par cette objection. Mais c'est néanmoins à cause d'elle que nous n'avons pas voulu faire de cette réaction une méthode de dosage.

Il faut considérer cette méthode comme un procédé de triage rapide permettant d'éliminer avec certitude les produits exempts de diacétyle, car si la réaction ne fournit aucune coloration, c'est bien qu'on n'a pas trace de diacétyle dans le distillat.

Si au contraire la réaction est positive et si on conservait un doute quelconque, on a plusieurs autres moyens de caractériser le diacétyle avec certitude.

$$
\text { ** * }
$$

\section{CARACTÉRISATION PRÉCISE DU DIACÉTYLE PAR LA PHENYLHYDRAZINE.}

On sait que les sucres aldéhydiques et les sucres cétoniques ont la propriété de donner, avec la phénylhydrazine, des composés $q u$ 'on appelle phénylhydrazones; ces substances peuvent se condenser avec une nouvelle molécule de phenylhydrazine; on obtient alors des diphénylhydrazones ou osazones qui sont des substances très caractéristiques par la forme de leurs cristaux, leur point de fusion, etc...

Mais cette propriété n'est pas spéciale aux sucres réducteurs. Certains corps polycétoniques donnent des réactions analogues.

(On sait par exemple que les acides $\beta$ dicétoniques conduisent de cette manière à des chaînes pyrazoloniques qui ne sont d'ailleurs pas des osazones vraies et que c'est là le point de départ de la fabrication industrielle de l'antipyrine).

Les dicétones $\alpha$ (donc le diacétyle) donnent aussi comme les sucres réducteurs des phénylhydrazones et des osazones.

Une première molécule de phénylhydrazine se fixe sur un groupe 
cétonique du diacétyle pour donner une phénylhydrazone de formule :

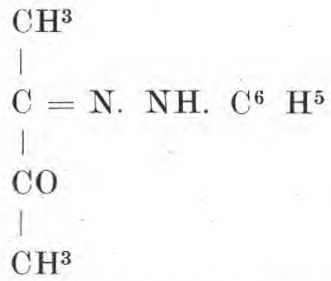

Ce corps se forme aisément à froid, même en solution aqueuse.

En présence d'un excès de phénylhydrazine et au bain-marie bouillant, une deuxième molécule de phénylhydrazine se fixe sur le deuxième $\mathrm{CO}$ du diacétyle pour donner la diacétylosazone suivant la réaction :

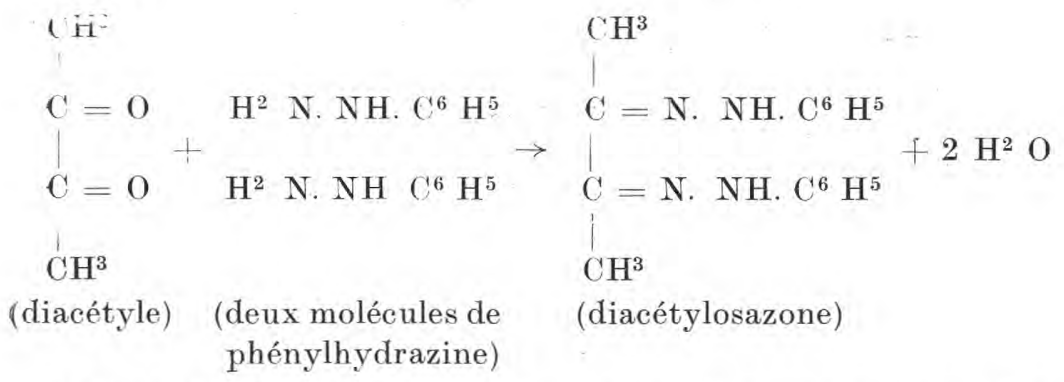

Notons bien que les osazones vraies ne se forment que dans le cas des sucres aldéhydiques ou cétoniques, et dans le cas des dicétones $\alpha$. Donc parmi les substances volatiles ou entraînables à la vapeur d'eau, seul le diacétyle (et ses homologues supérieurs dicétoniques en $\alpha$ ) donne des osazones. C'est là un caractère de spécificité sur lequel il était bon d'insister.

Mais une remarque très importante s'impose: d'une manière générale dans l'utilisation de la phénylhydrazine on ne s'arrête pas au stade phénylhydrazone et on cherche toujours à avoir l'osazone en raison de caractères cristallographiques souvent très nets.

Or, dans le cas du diacétyle, l'expérience nous a montré que la phénylhydrazone présente une forme cristalline très caractéristique avec mâcles nettes en croix de St-André, alors que l'osazone ne donne que de petits cristaux sans groupements caractéristiques.

Donc, au point de vue de l'identification du diacétyle, la phénylhydrazone nous paraît supérieure à l'osazone et c'est à cette technique que nous nous sommes arrêtés. (D'ailleurs la préparation de l'hydrazone est plus facile et plus rapide que celle de l'osazone.)

Voici comment nous préparons la diacétylphénylhydrazone : Dans un tube à essai on introduit 5 cc environ de solution contenant 
du diacétyle. (Il est conseillé d'opérer sur une solution diluée pour avoir de plus beaux cristaux. Si on pense que la solution de diacétyle est beaucoup plus riche que 1/1.000, la diluer pour rester en dessous de cette concentration.)

Ajouter 0 cc 5 d'une solution aqueuse de phénylhydrazine à $1 \%$.

On remarquera que nous évitons l'usage de l'acide acétique généralement utilisé dans ce genre de réactions. Cet acide, en effet, provoque une précipitation brutale conduisant à des cristaux très fins et moins beaux. C'est pourquoi nous restons en milieu aqueux.

On laisse la réaction s'opérer à froid. Au bout de quelques instants il se développe une belle coloration jaune. Cinq minutes plus tard le liquide se trouble. Il se forme un précipité de diacétylphénylhydrazone qui se dépose en 10-15 minutes.

La sensibilité de cette réaction est notable: on obtient un abondant précipité dans une solution de diacétyle au $1 / 1.000$, et des précipités très nets à des dilutions plus grandes.

Mais ce qui fait surtout l'intérêt de cette réaction, c'est, avonsnous dit, la spécificité des caractères cristallographiques du précipité. (Voir ci-après les micro-photographies que nous avons obtenues de ces cristaux mâclés.)

Le point de fusion de la diacétylphénylhydrazone déterminé par nos soins, est de $130^{\circ}$ (certains auteurs donnent $133^{\circ}$ ).

Remarque: Dans le cas de solutions très diluées il ne se forme pas de précipité mais une coloration jaune (due à la solubilité de l'hydrazone). On laisse alors la réaction se produire plus longtemps et on extrait par l'éther la phénylhydrazone formée. On opère ainsi :

5 cc du liquide à essayer sont, comme ci-dessus, additionnés de 0 cc 5 de solution aqueuse de phénylhydrazine à $1 \%$. On abandonne une heure. On ajoute 2 ce d'éther et on agite. A l'aide d'une pipette compte-gouttes on prélève l'éther que l'on porte dans un autre tube. On ajoute $2 \mathrm{cc}$ d'alcool à $96^{\circ}$. Ce mélange est repris avec la même pipette. On pose la pointe de cette pipette sur une lame de verre et on laisse le liquide s'écouler très lentement de lui-même (sans appuyer sur le caoutchouc du compte-gouttes). L'évaporation se produit pendant l'écoulement et on réalise ainsi une cristallisation très lente à froid. Les cristaux ainsi obtenus sont en tout point semblables à ceux obtenus par précipitation directe d'une solution à $1 / 1.000$.

Cette technique permet la formation de phénylhydrazone à partir d'une solution de diacétyle au 1/20.000. (Nous verrons d'ailleurs que la méthode de dosage décrite ci-après permet d'aller avec sécurité à des dilutions de 1/100.000.) 

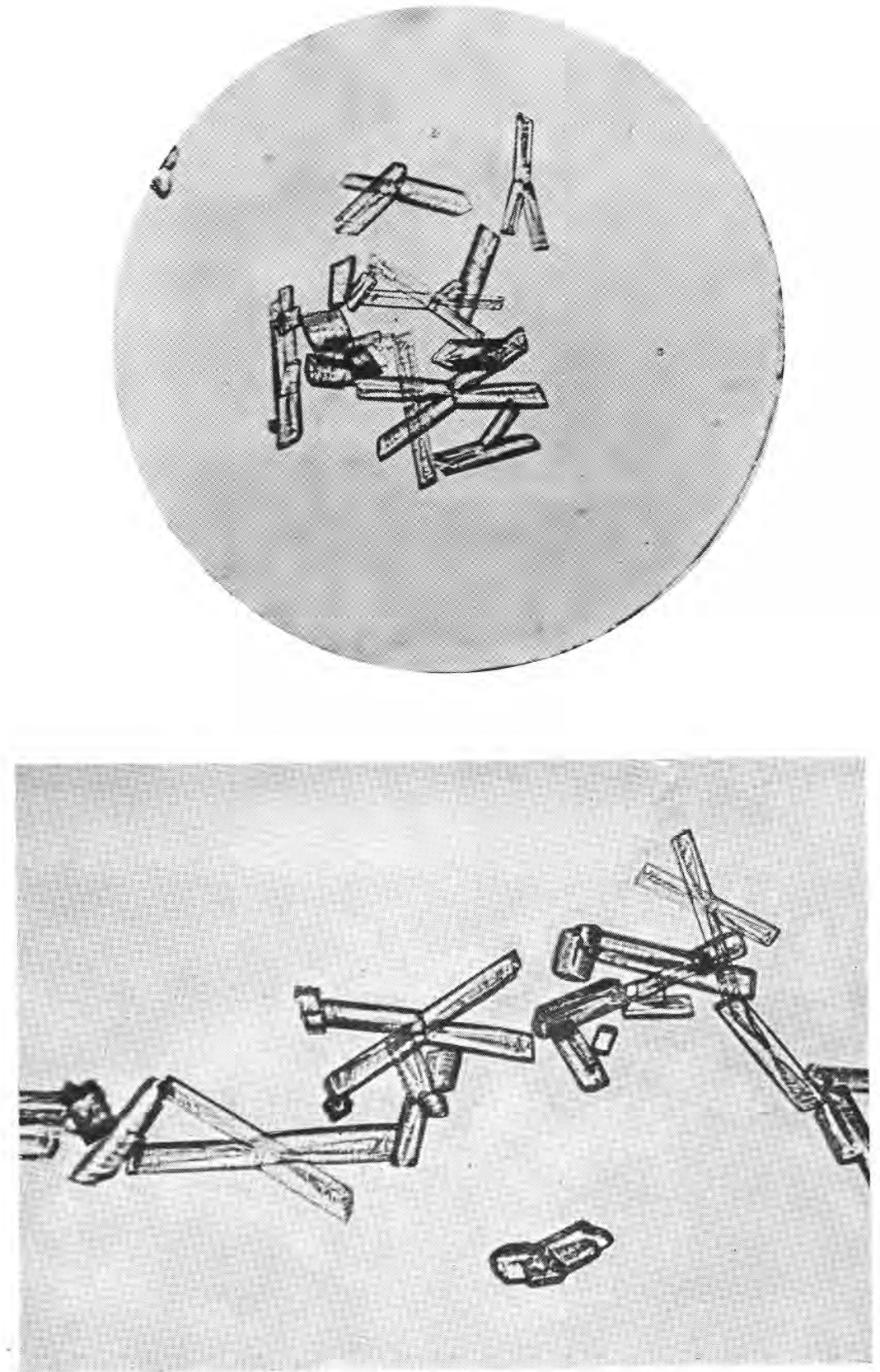

Photographies "Fermiers Réunis " Cristaux de diacétylphénylhydrazone 
Cette technique constitue un procédé de contrôle précieux dans le cas où les autres méthodes de recherche auraient laissé un doute sur la présence éventuelle du diacétyle.

La formation de cette hydrazone, ses caractères cristallographiques et son point de fusion constituent des critères absolument sûrs.

$$
*^{*} *
$$

Une dernière méthode de recherche qualitative et d'identification nous a été suggérée par l'étude des composés d'addition des dicétones $\alpha$ et des orthodiamines aromatiques.

Comme cette méthode est en même temps celle que nous avons retenue pour le dosage colorimétrique du diacétyle, nous l'étudierons ci-après.

$$
\begin{gathered}
* * * \\
\text { TROISIEMME PARTIE }
\end{gathered}
$$

\section{TROISIËME PARTIE}

\section{NOUVEAU PROCÉDÉ DE DOSAGE DU DIACÉTYLE}

\section{Principe.}

On sait qu'un groupement cétonique R.CO peut se condenser avec un groupement aminé $\mathrm{R}^{1} \mathrm{NH}^{2}$ appartenant à une autre molécule, pour donner, avec élimination d'eau, des corps de la forme

$$
\text { R.C. }=\text { N.R }{ }^{1}
$$

Dans le cas des dicétones $\alpha$, comme le diacétyle, chacun des deux groupements CO peut participer à une réaction de cette nature à condition que les deux groupements aminés que l'on fera intervenir soient également situés sur des carbones voisins. Si on fait appel à une diamine aromatique (et il le faut puisque c'est le meilleur moyen d'espérer obtenir un produit de condensation coloré) les deux groupements aminés devront être en ortho l'un par rapport à l'autre.

D'où l'idée de condenser le diacétyle avec une orthodiamine aromatique (le corps obtenu sera une quinoxaline).

Dans le cas le plus simple, si on s'adresse à l'orthodiamidobenzène on aura une réaction de la forme :

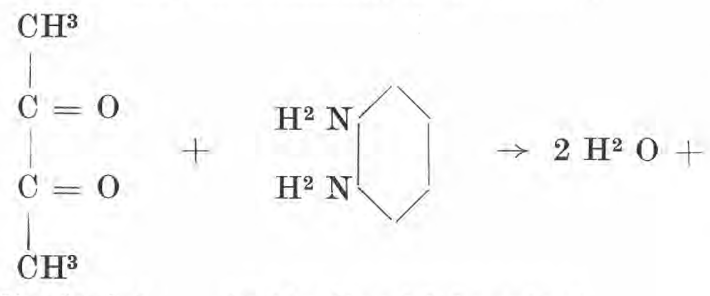

(diacétyle) (orthodiamidobenzène)

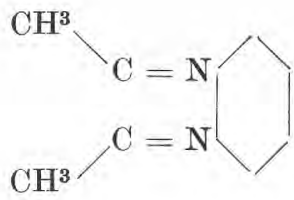

(quinoxaline) 
Cette quinoxaline est une substance colorée qui se prête bien à des dosages colorimétriques.

Malheureusement les orthophénylènediamines ne se trouvent pas couramment dans le commerce et leur préparation est en général difficile.

Toutefois si on s'adresse à l'homologue supérieur de l'orthodiamidobenzène que l'on vient de prendre comme exemple, autrement dit si on fait appel à une diamine du toluène au lieu d'une diamine du benzène, la préparation est beaucoup plus facile. C'est la raison pour laquelle nous nous sommes arrêtés au choix d'une toluilène-diamine.

Remarque: Il faut veiller bien entendu à ce que les deux groupes $\mathrm{NH}^{2}$ soient contigus, c'est-à-dire en ortho l'un par rapport à l'autre. On a donc deux combinaisons possibles : soit faire appel à l'ortho-métatoluilènediamine :

$\mathrm{CH}^{3}$

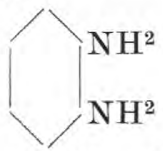

soit s'adresser à la métaparatoluilènediamine :

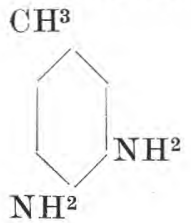

C'est cette dernière que nous avons choisie pour des raisons de commodité de préparation. (Les deux autres combinaisons possibles se ramènent aux deux combinaisons ci-dessus.)

Avant de décrire la réaction il nous faut done montrer comment on prépare cette toluilènediamine que l'on ne trouve pas couramment dans le commerce.

\section{Préparation de la Méta-Para-Toluilène-Diamine.}

$I^{0}$ Acétylation de la paratoluidine. - On part de la paratoluidine (100 gr. environ) que l'on acétyle pour bloquer la fonction amine :
$\mathrm{CH}^{3}$
$+\mathrm{CH}^{3}-\mathrm{COO}-\mathrm{CO}-\mathrm{CH}^{3} \rightarrow \mathrm{H}^{2} \mathrm{O}+\mathrm{CH}^{3} \mathrm{COOH}+$
$\mathrm{NH}^{2}$
$\mathrm{CH}^{3}$
1

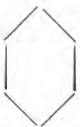
(parato-
(anhydride acétique)
NH. CO. $\mathrm{CH}^{3}$
luidine) 
On utilise environ le même poids d'anhydride acétique (100 gr.).

La toluidine se dissout d'abord dans l'anhydride. Il y a échauffement, puis le liquide se prend en masse par cristallisation. On essore au Büchner, on lave à l'eau et l'on recristallise dans l'alcool à $90^{\circ}$.

Cette acétylation a pour but de protéger la fonction amine pendant l'opération suivante.

$2^{\circ}$ Nitration de la toluidine acétylée. - Le produit obtenu est projeté par petites fractions de 1 à 2 grammes dans 4 fois son poids d'acide nitrique fumant $(\mathrm{d}=1,48)$.

La température ne doit pas dépasser $30^{\circ}$.

Le rôle de l'acide nitrique est de provoquer la fixation d'un $\mathrm{NO}^{2}$ contigu au groupement amine (momentanément bloqué par acétylation). On obtient done :

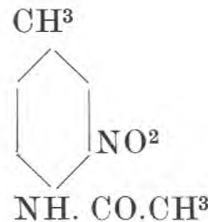

Le liquide ainsi obtenu est versé dans un grand excès d'eau froide. La nitrotoluidine précipite. Ce précipité est purifié par dissolution dans l'eau bouillante et recristallisation à froid. On obtient ainsi de fines aiguilles jaunes qu'on redissout dan. le mcins d'alcool possible.

$3^{\circ}$ Libération du groupement $\mathbf{N H}^{2}$ bloqué. - La nitrotoluidine obtenue a toujours son radical $\mathrm{NH}^{2}$ bloqué par acétylation. On le libère en traitant le produit à l'ébullition par une quantité de potasse un peu supérieure à la quantité théorique.

En refroidissant il se précipite des cristaux rouges (point de fusion $116^{\circ}$ ) de nitrotoluidine pure de formule:

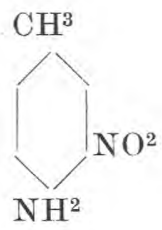

$4^{\circ}$ Formation du deuxième groupement aminé. - Les cristaux précédents, essorés, sont recouverts d'acide chlorhydrique concentré. On ajoute deux fois le poids du produit de grenaille d'étain. L'ensemble s'échaufie. L'hydrogène naissant produit, transforme le $\mathrm{NO}^{2}$ en $\mathrm{NH}^{2}$. 
La Métaparatoluilènediamine est formée (à l'état de chlorhydrate). Il ne reste plus qu'à la débarrasser du chlorure d'étain (pour cela on étend d'eau et fait passer un courant de $\mathrm{H}^{2} \mathrm{~S}$. On filtre, on évapore à sec au bain-marie). Il faut enfin libérer la base libre de son chlorhydrate.

$5^{\circ}$ Extraction de la toluilènediamine pure.- Le résidu de l'évaporation est mélangé avec de la chaux pulvérisée et distillé à sec dans un long tube de Pyrex. Cette opération libère la diamine de son sel. On obtient:

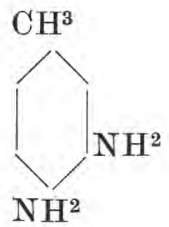

La base que nous avons ainsi obtenue distille en un liquide huileux, réfringent, qui cristallise en écailles fusibles à $88^{\circ} 5$.

\section{Réaction qualitative entre la Toluilènediamine et le Diacétyle.}

Prendre $10 \mathrm{ec}$ de la solution à essayer dans un tube à essai.

$\mathrm{Y}$ verser 0 ec 5 d'une solution aqueuse de métaparatoluilènediamine à $1 \%$. Agiter.

Ajouter ensuite lentement, à l'aide d'une pipette appuyée contre la paroi du tube, $10 \mathrm{cc}$ d'acide sulfurique concentré. Il se développe une belle coloration jaune due à la quinoxaline formée par la condensation des deux substances.

On incline et redresse le tube plusieurs fois pour réaliser un bon mélange et on abandonne pendant une heure, temps au bout duquel la coloration a atteint sa valeur maxima.

Sensibilité : La coloration est encore nettement appréciable avec une solution de diacétyle au 1/100.000. Cette méthode permettra donc la recherche de traces de diacétyle.

Remarque : Il convient de s'assurer par un essai à blanc sans diacétyle, que la toluilènediamine ne donne aucune coloration en présence de l'acide sulfurique. En fait, nous n'avons jamais eu de coloration en l'absence de diacétyle.

\section{Application au dosage colorimétrique du Diacétyle.}

I ${ }^{0}$ Utilisation, comme témoin, d'une solution type de diacétyle. - Nous avons préparé du diacétyle chimiquement pur en suivant les indications données plus haut. Nous avons réalisé des solutions titrées de diacétyle (dans l'eau). 
Puis nous avons effectué la réaction précédente à la fois sur les olutions titrées de diacétyle et sur les solutions inconnues à doser. En principe nous partons comme solution de base d'une solution de diacétyle au 1/5.000.

Pour cette concentration la quinoxaline formée présente une teinte d'une bonne intensité moyenne.

Si la liqueur à essayer donne une coloration plus intense, on la diluera au préalable de manière à la rapprocher du témoin (les dosages colorimétriques ne sont valables que si les deux liquides à comparer sont de richesse voisine).

En revanche, si la liqueur à essayer donne une coloration plus íaible que le témoin, on fera un nouveau témoin au 1/10.000, ou au $1 / 20.000$, etc...

\section{$2^{\circ}$ Utilisation d'une gamme de témoins artificiels. -} La technique qui vient d'être exposée nécessiterait de posséder du diacétyle pur et de refaire à chaque essai des solutions témoins convenablement diluées (et de leur faire subir la réaction).

Il est possible de remplacer ces solutions de diacétyle pur par une solution définitive et stable d'une matière colorée présentant la teinte exacte de la quinoxaline qui résulte de la réaction entre le diacétyle et la toluilènediamine.

Le réactif reproduisant le mieux la tonalité de la quinoxaline est le bichromate de potasse en solution aqueuse.

On commence par faire une solution mère de bichromate de potasse à $1 \%$ dans l'eau distillée. Puis on prendra un certain nombre de cc $(\mathrm{N})$ de cette solution et on les diluera à $200 \mathrm{cc}$ avec de l'eau distillée. On comparera la teinte de ces témoins à la teinte obtenue avec la liqueur à essayer, dans les conditions décrites plus haut.

Voici la correspondance que nous avons établie entre les témoins de bichromate et le taux de diacétyle ayant fourni une liqueur quinoxalinique de même teinte:

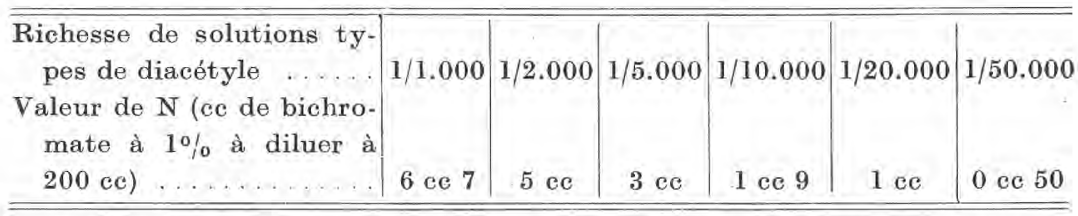

Bien entendu cette échelle pourrait être étendue à des concentrations plus fortes; mais nous avons constaté que le besoin s'en fait rarement sentir et que de toute façon il est préférable de diluer une liqueur trop riche en diacétyle afin de ramener la quinoxaline formée à une intensité de coloration plus propice à l'exécution d'un bon dosage. 


\section{Remarques :}

$1^{\circ} \mathrm{Il}$ est à conseiller, pour la réussite des titrages colorimétriques en général, de s'inspirer des excellentes directives données récemment par R. Doirque (Bull. Société Chimie Biolog. Octobre 1935, page 1304).

$2^{\circ}$ Cette méthode de dosage est d'une grande simplicité (seule la préparation de la toluidènediamine est une légère complication). Elle permet des titrages rapides, sûrs. Nous l'avons appliquée à maintes reprises et pensons qu'elle est susceptible de convenir à tous les besoins relevant de la laiterie, c'est-à-dire, recherche et dosage du diacétyle dans les cultures de ferments, la crème, le beurre.

Nous allons voir maintenant comment on l'applique dans la pratique et à quels résultats elle conduit.

(A suivre.)

\section{DIRECTIVES POUR L'EXÉCUTION DU CONTROLE BACTÉRIOLOGIQUE DU LAIT (1)}

Ces directives ont été établies à l'occasion du concours de l'Exposition d'alimentation du Reich en 1935 par le Privat-Docent $\mathrm{D}^{\mathrm{r}}$ K. J. Demeter, Chef de la Section bactériologique de l'ÉtaWlissement d'essais et de recherches laitières de l'Allemagne du Sud à Weihenstephan, École Polytechnique de Munich.

$$
*^{*} *
$$

Le nouveau règlement du concours réservé au lait et aux produits laitiers a déjà fait l'objet d'un travail fondamental du $\mathrm{D}^{\mathrm{r}} \mathrm{Hans}$ Adalbert SchWEIGART, ehef de section principal au Commissariat du Bétail, Lait et Graisse.

Il est exact que l'on peut considérer comme révolutionnaires les nouveaux procédés qui sont décrits dans le règlement pour l'estimation et le contrôle des produits laitiers. Il est encore trop tôt pour entreprendre la justification de la réforme profonde apportée aux méthodes d'examen du lait. Il faut plutôt considérer ce règlement comme un premier essai et en particulier admettre que la nature, le nombre et l'importance accordée aux différents essais bactériologiques que l'on doit exécuter sur le lait, ne sont pas détinitivement fixés ; il est même probable qu'ils seront modifiés dans l'avenir. Il faut done dissiper dès maintenant les scrupules de ceux qui estiment que tel essai est trop sévère, que tel autre est insuffi-

(1) Deutsche Molkerei-Zeitung (anciennement Süddeutsche Molkerei-Zeitung), Kempten im Allgäu, novembre 1934. Travail complété et mis à jour. 